\title{
Single electron emission in two-phase xenon with application to the detection of coherent neutrino-nucleus scattering
}

\section{ZEPLIN-III Collaboration}

E. Santos, ${ }^{1,2}$ B. Edwards, ${ }^{3}$ V. Chepel, ${ }^{1}$ H.M. Araújo, ${ }^{2}$ D.Yu. Akimov, ${ }^{4}$ E.J. Barnes, ${ }^{5}$ V.A. Belov, ${ }^{4}$ A.A. Burenkov, ${ }^{4}$ A. Currie, ${ }^{2}$ L. DeViveiros, ${ }^{1}$ C. Ghag, ${ }^{5}$ A. Hollingsworth, ${ }^{5}$ M. Horn, ${ }^{2}$ G.E. Kalmus, ${ }^{3}$ A.S. Kobyakin, ${ }^{4}$ A.G. Kovalenko, ${ }^{4}$ V.N. Lebedenko, ${ }^{2}$ A. Lindote, ${ }^{1,3}$ M.I. Lopes, ${ }^{1}$ R. Lüscher, ${ }^{3}$ P. Majewski, ${ }^{3}$ A.St J. Murphy, ${ }^{5}$ F. Neves, ${ }^{1,2}$ S.M. Paling, ${ }^{3}$ J. Pinto da Cunha, ${ }^{1}$ R. Preece, ${ }^{3}$ J.J. Quenby, ${ }^{2}$ L. Reichhart, ${ }^{5}$ P.R. Scovell, ${ }^{5}$ C. Silva, ${ }^{1}$ V.N. Solovov, ${ }^{1}$ N.J.T. Smith, ${ }^{3}$ P.F. Smith, ${ }^{3}$ V.N. Stekhanov, ${ }^{4}$ T.J. Sumner, ${ }^{2}$ C. Thorne $^{2}$ \& R.J. Walker ${ }^{2}$

${ }^{1}$ LIP-Coimbra \& 8 Department of Physics of the University of Coimbra, Portugal

${ }^{2}$ High Energy Physics group, Blackett Laboratory, Imperial College London, UK

${ }^{3}$ Particle Physics Department, STFC Rutherford Appleton Laboratory, Chilton, UK

${ }^{4}$ Institute for Theoretical and Experimental Physics, Moscow, Russia

${ }^{5}$ School of Physics 83 Astronomy, University of Edinburgh, UK

E-mail: e.santos10@imperial.ac.uk

ABSTRACT: We present an experimental study of single electron emission in ZEPLIN-III, a two-phase xenon experiment built to search for dark matter WIMPs, and discuss applications enabled by the excellent signal-to-noise ratio achieved in detecting this signature. Firstly, we demonstrate a practical method for precise measurement of the free electron lifetime in liquid xenon during normal operation of these detectors. Then, using a realistic detector response model and backgrounds, we assess the feasibility of deploying such an instrument for measuring coherent neutrino-nucleus elastic scattering using the ionisation channel in the few-electron regime. We conclude that it should be possible to measure this elusive neutrino signature above an ionisation threshold of $\sim 3$ electrons both at a stopped pion source and at a nuclear reactor. Detectable signal rates are larger in the reactor case, but the triggered measurement and harder recoil energy spectrum afforded by the accelerator source enable lower overall background and fiducialisation of the active volume.

KEYWORDS: xenon detectors, single electron emission, electroluminescence, ZEPLIN-III, coherent neutrino scattering, reactor antineutrinos, stopped pion source 


\section{Introduction}

ZEPLIN-III is a two-phase xenon detector designed for the direct detection of WIMP dark matter $[1,2]$. The two-phase technique [3-5] produces two different signals for each particle interacting within the active volume, one from primary scintillation in the liquid (S1) and the other from electroluminescence in the gas phase (S2). This second signal is a measure of the amount of ionisation drifted from the interaction site by application of an electric field and subsequently emitted from the liquid surface. The scintillation and ionisation yields differ for electron and nuclear recoils, providing a physical basis for the discrimination between interaction types. Since discrimination requires both signals, in typical WIMP dark matter searches the energy threshold is determined by the less sensitive S1 response channel.

The ionisation signal is measured through cross-phase emission of electrons extracted from tracks in the liquid into the xenon vapour above it. Here, they are accelerated by a strong electric field and induce secondary scintillation (electroluminescence) in the xenon vapour. The photon yield is a linear function of the field $E$ and can be parametrised by $N_{p h}=(a E+b n) x$, where $x$ is the thickness of the gas layer, $n$ is the number density of xenon atoms and the coefficients $a$ and $b$ were determined experimentally for saturated xenon vapour in Ref. [6]. For typical ZEPLIN-III operational parameters $(E=7-8 \mathrm{kV} / \mathrm{cm}$ in the gas at 1.6 bar, $x \sim 4 \mathrm{~mm}$ ), some 300 photons are produced by a single electron emitted from the liquid.

The possibility of exploiting the sensitivity of the ionisation channel down to the single electron level (sub-threshold in S1) has been pointed out in the context of coherent neutrinonucleus scattering in 2004 [7]. This motivated a programme based on two-phase argon for nuclear reactor monitoring [8]. Interest in this experimental technique has now also extended to searches for light WIMPs [9]. With this type of application in mind, we first characterised the single electron signature using ZEPLIN-II data [10] and in work with a smaller prototype chamber operating in a surface laboratory [11].

This article is organised as follows. In Section II we discuss the single electron signature and likely production mechanisms based on analyses of independent datasets acquired under different conditions. This leads to the demonstration of a very practical application: the determination of the free electron lifetime in the liquid phase by using WIMP-search data only. This could be especially relevant for next-generation WIMP experiments based on the noble liquids. In Section III we assess the feasibility of using the ionisation channel down to single electron level to measure the coherent elastic scattering of neutrinos off nuclei, a Standard Model process not yet observed. We include realistic signal characteristics and a background of single electron pulses which might be produced by the mechanisms identified in the preceding section. Previous studies have typically assumed idealised response models. 

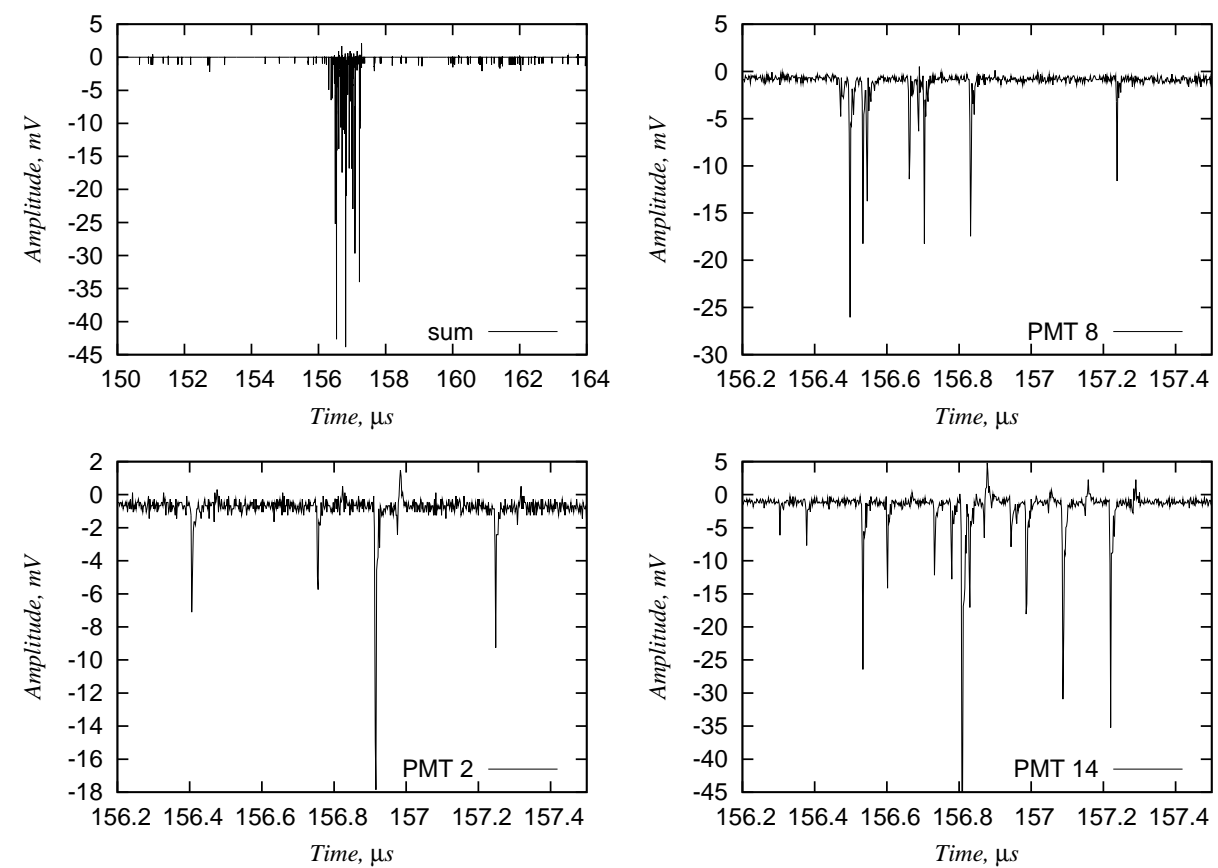

Figure 1. A single electron signal in ZEPLIN-III (sum waveform shown top left; channel waveforms containing detected photoelectrons are zoomed in).

\section{Single electrons in ZEPLIN-III}

Single electron signals were studied using four independent datasets: two WIMP-search exposures (of several months' duration) and two dedicated runs. The main difference between the two types of dataset is the origin of the trigger for data acquisition (DAQ). During dark matter data-taking, the trigger function was derived from S1 or S2 pulses in the detector, recording $\pm 18 \mu$ s waveforms centred around the trigger point. In the dedicated runs, on the other hand, the DAQ was triggered externally with a pulse generator at a fixed (high) repetition rate, independently of any signals in the detector. In this instance the acquired waveforms were $256 \mu$ s long, which exceeds significantly the maximum ionisation drift time in the chamber $(\sim 14.5 \mu \mathrm{s})$. We begin by describing the set-up and results from the first dedicated single-electron run (hereafter 'DSER'), which underwent the most extensive analysis. Key parameters for this and other datasets are summarised in Table I.

\subsection{Dedicated single electron run}

A total live time of $161.4 \mathrm{~s}$ was recorded during the DSER acquisition over a 2-day period after the completion of the first science run (FSR) in 2008. During that time, the xenon pressure in the detector remained stable at 1.6 bar and $E=7.6 \mathrm{kV} / \mathrm{cm}$ in the gas. The signals from each of the 31 PMTs were digitised with 2 ns sampling and 8-bit resolution over the $256 \mu$ s timelines. The DAQ system was forced to trigger at the maximum rate for this configuration $\left(18 \mathrm{~s}^{-1}\right)$ by an external pulse generator. The raw waveform data were reduced with the ZE3RA pulse analysis algorithms [12], using similar parameters to those 
Table 1. Single electron rates in ZEPLIN-III

\begin{tabular}{l|c|cc|c|c}
\hline \hline Run & $\begin{array}{c}\text { live time } \\
\text { seconds }\end{array}$ & $\begin{array}{c}\text { drift field } \\
\mathrm{kV} / \mathrm{cm}\end{array}$ & $\begin{array}{c}\text { cath. wire } \\
\mathrm{kV} / \mathrm{cm}\end{array}$ & $\begin{array}{c}\text { event rate } \\
\mathrm{s}^{-1}\end{array}$ & $\begin{array}{c}\text { SE rate } \\
\mathrm{s}^{-1}\end{array}$ \\
\hline DSER & 161 & 3.8 & 60 & 6.8 & $5.7^{*}$ \\
FSR & 194 & 3.8 & 60 & 6.8 & $\sim 20$ \\
SSR & 10.2 & 3.4 & 38 & 0.45 & $\sim 1$ \\
Cs-137 & 37 & 3.4 & 29 & 145 & $118^{*}$ \\
\hline \hline
\end{tabular}

$\dagger$ Electric field in the liquid xenon away from cathode.

$\ddagger$ Higher energy background above normal trigger threshold (S2>4 electrons).

* $20 \mu$ s inhibit period

adopted for the FSR data, except that a Fourier transform digital filter was applied to the waveforms to remove coherent noise induced by the pulse generator. The reduced data were then searched for photoelectron clusters, characteristic of single electron emission, at a minimum of 3-fold coincidence in the PMTs, with a pulse amplitude threshold of 4 times the rms noise in each waveform. An example of such cluster is shown in Figure 1. In this particular analysis, the number of single photoelectron pulses in a cluster is counted by the number of threshold crossings individually in each channel using a constant 50ns integration time. A channel-by-channel correction is then applied to account for the dead-time effect thus introduced (a constant-rate Poisson process is assumed).

The following selection cuts are applied in this analysis: i) no pulses present in the preceding $20 \mu \mathrm{s}$ in any waveform; this is greater than the ionisation drift time for the full liquid depth and ensures that selected events are not induced directly by a previous energy deposition in the liquid xenon; ii) the reconstructed $x, y$ position of the event must be within the central $60 \mathrm{~mm}$ radius of xenon (1.3 $\mathrm{kg}$ active mass); this allows the use of a simple centroid position reconstruction algorithm and ensures uniform light collection.

Figure 2 (left) shows the size distribution of photoelectron counts in single electron clusters for this dataset. The histogram was fitted by exponential and Gaussian components, resulting in a mean number of $28.3 \pm 0.3$ (stat) photoelectrons per pulse. The very large peak-to-valley ratio of the distribution confirms the excellent sensitivity to these pulses. The observed single electron event rate was $5.7 \mathrm{~s}^{-1}$. The $x, y$ spatial distribution of these events, shown in Figure 3, is relatively uniform within the selected volume, although a bias is observed at larger radii. This is attributed to a small and well-understood tilt of the detector of a few mrad, which affects the thickness of the gas layer (and, to a lesser degree, the electric field strength), causing a systematic variation in the detection efficiency at large radii.

\subsection{The WIMP science runs}

The first science run of ZEPLIN-III acquired WIMP-search data for 83 days. Details of the data acquisition and primary dark matter analyses are described elsewhere [2, 13-15]. Two populations of small S2-like pulses were observed in the waveforms. One follows large S1 

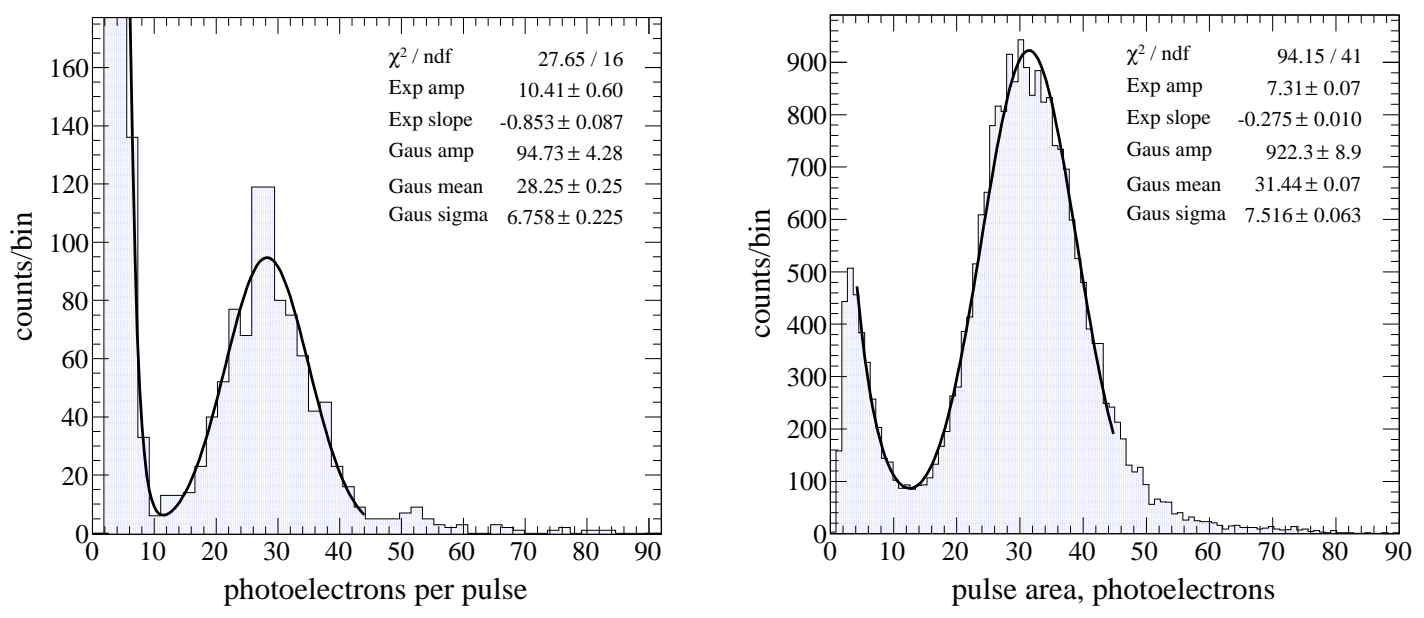

Figure 2. Single electron pulse size distributions in the first science run configuration of ZEPLINIII. LEFT: Number of photoelectrons per cluster obtained with the dedicated DSER dataset by photoelectron counting (dead-time corrected), as described in §2.1. Right: Size distribution of post-S1 pulses in the FSR science data, obtained by pulse area integration and converted subsequently into photoelectron numbers $(\S 2.2 \cdot 1)$.

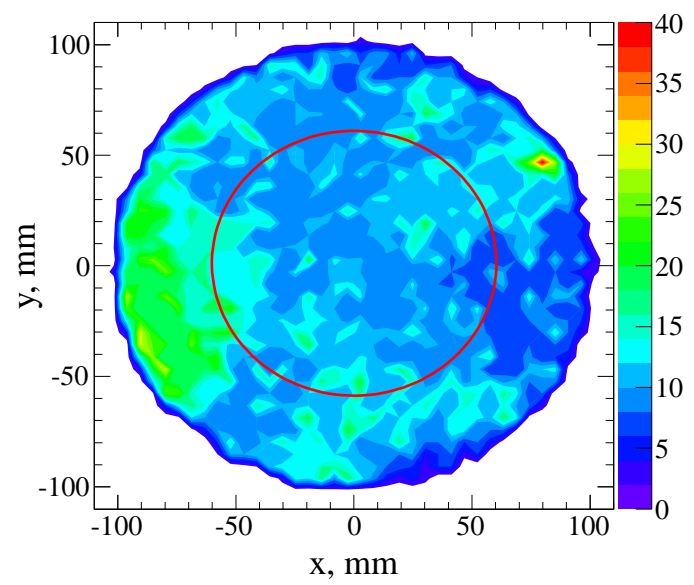

Figure 3. Centroid-reconstructed position of 'spontaneous' single electron pulses in the DSER dataset. This analysis extends to $60 \mathrm{~mm}$ radius. The bias observed at large radii is due to a small detector tilt which increases the gas layer thickness where an excess of events is observed.

or S2 signals and is clearly induced by the VUV luminescence [10]; this population allows for an accurate characterisation of single electron clusters. Another can be observed in the quiet pre-trigger region of the waveforms, before S1 pulses, and may be unrelated (at least directly) to energy depositions from interactions in the active volume; a 'spontaneous' rate 
can be measured for this population. ${ }^{1}$ We associate these clusters with those studied in the DSER dataset. We now treat both types of signal in turn.

\subsubsection{Photon-induced signals and their application}

In the FSR, a significant fraction of recorded events contained only an S1 pulse due to interactions below the cathode grid from $\beta$ and $\gamma$ backgrounds from the PMT array. A reverse electric field there does not allow electrons to drift to the sensitive volume and produce S2 signals. These otherwise clean waveforms provide the ideal dataset to search for the small single electron signals and to subsequently test the photoionisation production mechanism suggested previously [10]. The FSR waveforms were processed by the same data reduction algorithms as those implemented for the dark matter search data, but with some reduction parameters optimised for the clustering of even smaller S2-like signals.

Instead of the photoelectron counting method employed with the DSER, in this analysis the areas of clustered pulses were integrated in each channel; these were then converted into photoelectron numbers by using the single photoelectron response for each phototube determined by the method described in Ref. [12]. The pulse area distribution shown in Figure 2 (right) yields a mean $31.4 \pm 0.07$ (stat) photoelectrons per electron, which is only $\approx 10 \%$ higher than the value determined by the counting method employed with the DSER dataset. The standard deviation is also slightly larger in this instance (which is expected for a dataset acquired over a much longer period and which includes uncertainties in the single photoelectron response determined for each channel), but neither is far from the Poisson limit of $\approx 5.5$ photoelectrons. As had been observed in ZEPLIN-II, the frequency of these events is proportional to the number of VUV photons in the preceding S1 pulse, which points to a photoionisation origin [10].

The distribution of time elapsed between S1 and the single electron cluster is shown in Figure 4 for a typical day's data. The spike in event rate near $14.5 \mu$ s is due to photo-electric production by $\mathrm{S} 1$ photons incident on the cathode grid (we find its probability to be broadly consistent with the quantum efficiency of metals at VUV wavelengths). The exponential trend observed away from the cathode is consistent with an electron attachment explanation based on an independent lifetime measurement $(23.2 \pm 1.5 \mu \mathrm{s}$ for that day, obtained as explained below). This suggests that S1-induced electron emission might enable a good measurement of this parameter, provided that these electrons are uniformly distributed in the liquid depth. Both the spatial distribution of energy depositions in the chamber and the attenuation length for VUV light must be considered in determining whether the VUV flux from scintillation is constant across the liquid. The small vertical dimension of the chamber and the uniform horizontal distribution of this background ensure that this is indeed the case. The photon mean free path for photoionisation was estimated in Ref. [10] as $\sim \mathrm{km}$, so a uniform VUV flux implies a constant probability for electron appearance with depth, which translates to an exponential survival probability as observed in Figure 4.

This depth distribution should provide a measurement of the free electron lifetime in a rather robust and expedite way, relying solely on the science waveforms themselves (as in

\footnotetext{
${ }^{1}$ This terminology does not preclude a radiation-induced origin for these events; its use in quotation marks highlights that no event was detected within the maximum drift time of the time projection chamber.
} 


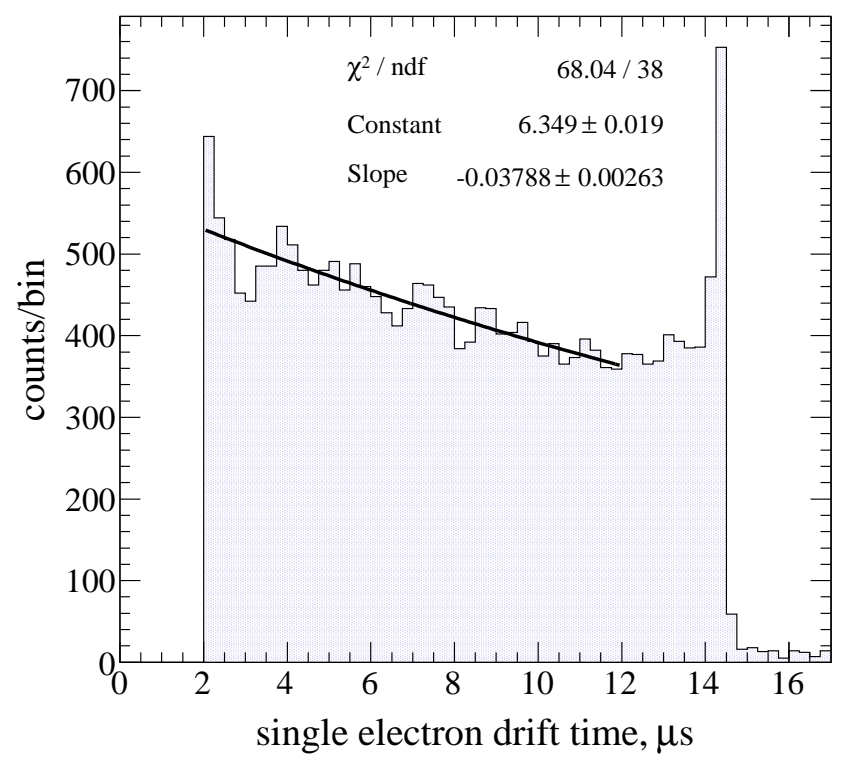

Figure 4. Drift time (i.e. depth) distribution of S1-induced single electron pulses for one day of FSR data. The cathode grid, located at $14.5 \mu \mathrm{s}$ drift time, produces an excess due to photo-electric emission from the stainless steel wires caused by the scintillation VUV photons. The first $2 \mu$ s were excluded since these are contaminated by PMT afterpulsing following large S1 pulses. The time constant of the exponential fit gives an electron lifetime in the liquid $\tau=26.4 \pm 2.0 \mu \mathrm{s}$.

this instance) - rather than on dedicated calibration datasets. Typically, this calibration is conducted by fitting the S2/S1 ratio as a function of drift time (S1-S2 time separation) in response to ${ }^{57} \mathrm{Co}$ or other external $\gamma$-ray sources. In this instance it is not straightforward to prevent (or correct for) saturation of the PMT readout for very large S2 pulses, which may easily contain upwards of $10^{5}$ photoelectrons. The very high VUV photon rates in the chamber may also affect the operation of these detectors in other, more subtle ways (e.g. photocathode charging [16]). The single electron signature provides a mechanism which involves only very small pulses which are anyway present in the data.

Figure 5 compares historical lifetime measurements with this method with those obtained from the daily calibration with ${ }^{57} \mathrm{Co}$ for both science runs of ZEPLIN-III. The agreement is very good in both cases. We anticipate that this new method may be especially useful for next-generation two-phase xenon and argon experiments, where routine irradiation of the large WIMP targets with external sources will be even harder to achieve. Even if not used on its own, this technique may be useful to assess any biases in the traditional measurement.

\subsection{2 'Spontaneous' signals in the science runs}

Another single-electron search was conducted in segments of the WIMP-search waveforms preceding S1 pulses, totalling $\sim 200$ s of live time. These events, like those found in the 


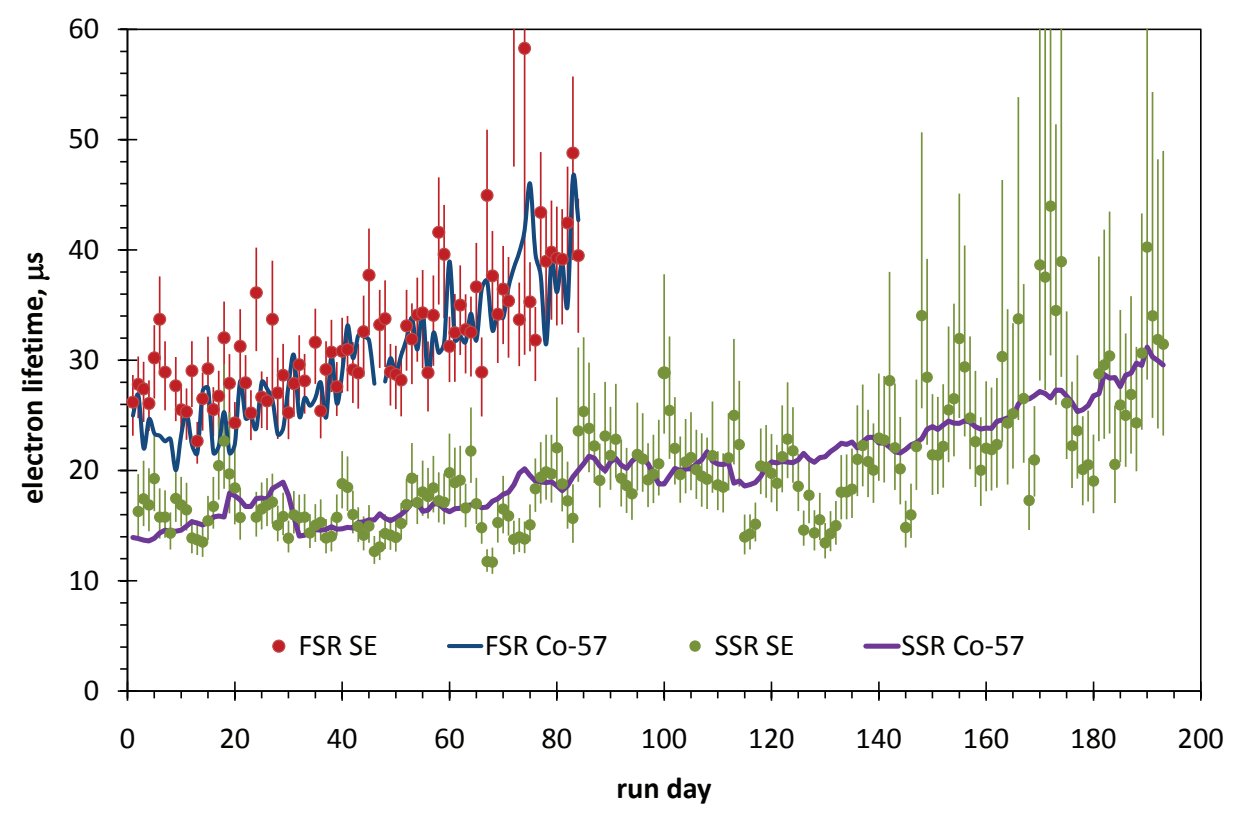

Figure 5. Historical evolution of the free electron lifetime in the liquid xenon measured from single electron signals (markers) and from the daily ${ }^{57} \mathrm{Co}$ calibration in the FSR and the first 200 days of the SSR (lines). The latter data were corrected independently for PMT saturation for large S2 signals but were not scaled otherwise.

DSER, have no traceable correlation with particle interactions in the detector. The rate for this population (within the $60 \mathrm{~mm}$ reconstructed radius) stabilised at $\sim 20 \mathrm{~s}^{-1}$ approximately one month into the FSR (starting out twice as high initially). This is higher than the rate of $5.7 \mathrm{~s}^{-1}$ measured in the DSER. However, we note that in this instance the length of waveform available for analysis $(16 \mu \mathrm{s})$ would not allow enforcing a $20 \mu$ s inhibit time from a candidate pulse.

The experiment was upgraded after the first run, with new PMTs with considerably lower radioactivity replacing the previous array, reducing the electron-recoil background of the experiment by a factor of 18 [17]. The >300-day second science run (SSR) started in mid 2010. In these data, we observed a very significant reduction in the 'spontaneous' component, to a level of $\sim 1 \mathrm{~s}^{-1}$. However, the reduction in background was not the only difference between the FSR and SSR datasets: the electrode voltage configurations were also different, producing a slightly lower electric field in the drift region (13\%) and significantly lower at the cathode wire surface (see Table I). Therefore, one cannot conclude for a background-related explanation for this reduction based solely on this observation.

A thorough study of the electric field dependence of 'spontaneous' emission was not possible prior to decommissioning of the experiment at the Boulby mine. Nevertheless, a short dedicated dataset had been acquired in a similar fashion to the DSER described previously after the end of the SSR data-taking, but this time with a ${ }^{137} \mathrm{Cs}$ source located above the detector; the energy deposition rate in $\gamma$-rays was some 300 times greater than 
background. The resulting 'spontaneous' rate was $118 \mathrm{~s}^{-1}$. Significantly, reanalyses of this dataset requiring clear $40 \mu \mathrm{s}$ and $60 \mu$ s periods preceding the single electron cluster (rather than the standard $20 \mu \mathrm{s}$ ) yielded lower rates of $55 \mathrm{~s}^{-1}$ and $44 \mathrm{~s}^{-1}$, respectively. We may conclude, therefore, that not only does the total 'spontaneous' rate depend very significantly on the rate of energy deposition in the target, but it also decreases with increasing time delay from those energy depositions.

\subsection{Causes and implications of 'spontaneous' electron emission}

This signal will provide a background for applications aiming to explore the ionisation channel in the few-electron regime, and it is therefore important to ascertain its (possibly multiple) causes. Amongst those we single out i) delayed emission due to lower-than-unity cross-phase extraction probability and ii) field emission from the cathode grid.

The correlation between the 'spontaneous' emission and the $\gamma$-ray event rate in the SSR and the ${ }^{137} \mathrm{Cs}$ datasets - acquired with similar field configurations - argues for a delayed emission mechanism, which is further supported by the decrease in rate for progressively longer inhibit periods. The cross-phase emission probability [18] was $83 \%$ under the FSR/DSER conditions and $66 \%$ in the SSR $/{ }^{137}$ Cs runs. Most electrons heated by the electric field to energies above the field-dependent surface potential barrier are emitted promptly into the gas phase. Those left on the surface quickly thermalise with the medium, but the most energetic ones can still be emitted as thermal electrons, albeit with lower probability [19]. Thermal emission is naturally curtailed by the finite electron lifetime in the liquid, which was $45.4 \mu \mathrm{s}$ for the ${ }^{137} \mathrm{Cs}$ dataset. The observed decrease in emission rate is broadly consistent with this lifetime. Under this model, the $\sim 3 \times$ discrepancy between the rates observed in the FSR and DSER datasets can be attributed to the longer inhibit period enforced in the latter case.

We also conclude that field emission from the stainless steel cathode wires $(100 \mu \mathrm{m}$ diameter, $1 \mathrm{~mm}$ pitch) can contribute to the spontaneous single electron rate to some degree. The field strength at the surface of the wires is actually comparable to the so-called 'applied breakdown field' which characterises the onset of macroscopic field emission in metals (see, e.g., [20, 21]). This breakdown field is determined by electrode irregularities and is some two orders of magnitude lower than the the critical breakdown field for the material, which is determined by its work function (although the latter is, in this instance, decreased by $0.67 \mathrm{eV}$ due to immersion in the liquid xenon [22]). The electrode voltage configuration changed between the first and second science runs; although the electric field strength in the liquid xenon bulk was only $13 \%$ lower in the SSR, the field near the top of the wires, where field lines connect with the anode, actually decreased from $60 \mathrm{kV} / \mathrm{cm}$ to $38 \mathrm{kV} / \mathrm{cm}$ [23] (this is mainly a consequence of the lower voltage applied to the cathode, which also establishes a reverse field region to a second wire grid located underneath it which shields the PMT input optics). The field dependence of the emission current is extremely steep $\left(\propto E^{2} e^{E}\right.$ in the Fowler-Nordheim model [24]) and, being a quantum tunnelling effect, no strict threshold can be defined. For example, the two emission sites studied in [21] (caused by an insulating particle on the metal surface and by a surface imperfection) would cause far higher rates than observed here, although no measurable emission would 
result from the perfect wires. Therefore the $50 \%$ lowering of the field near the surface of the wires between runs could easily explain a reduction in electron emission rates of several orders of magnitude and may contribute to a baseline rate $\lesssim 1 \mathrm{~s}^{-1}$.

Based on these findings, controlling single-electron emission will require a low background experimental set-up, achieving near-unity emission probability for hot electrons (i.e. electric fields $\gtrsim 5 \mathrm{kV} / \mathrm{cm}$ below the surface) and perhaps maintaining a lower electron lifetime (so as to limit the delayed emission of thermal electrons). The first (and possibly second) of these requirements will be challenging for detectors operating in surface laboratories. Field emission from the cathode is also identified as a viable production mechanism, but it is perhaps the more benign of the two since careful electrode design should be able to avert significant spontaneous rates.

\section{Sensitivity to coherent neutrino-nucleus scattering}

We now assess the feasibility of measuring coherent neutrino-nucleus scattering using only the low-threshold ionisation channel in a two-phase xenon detector, with ZEPLIN-III as the working example. Having characterised the response to single electrons and identified the foremost sources of background for this signature, we must consider the uncertain ionisation yield for nuclear recoils in liquid xenon, which has not been measured experimentally below a few keV. However, present data (see also, e.g., Fig. 5 in Ref. [25]) suggest that the energy required to extract the first ionisation electron with appreciable probability is likely to be $<1 \mathrm{keV}$, and maybe significantly less.

For lack of experimental data at very low energies we resort to extrapolating existing ionisation yields in an ad hoc manner. We adopt the yield curve obtained for the ZEPLINIII FSR [25] down to $4 \mathrm{keV}$, which is in good agreement with other data. This yields a maximum of $6.5 \mathrm{e} / \mathrm{keV}$ at that energy, below which it is forced to inflect and vanish for zero recoil energy along a $3^{r d}$ order polynomial. It takes $540 \mathrm{eV}$ to create the first ionisation electron in this parametrisation. (A more optimistic scenario was also considered which matched the former above $6 \mathrm{keV}$ but followed instead the last three data points from Ref. [26] below that energy; this curve peaks at $\approx 10 \mathrm{e} / \mathrm{keV}$ for $2.7 \mathrm{keV}$, with the first electron emitted at $350 \mathrm{eV}$. However, the conclusions of this study remained unchanged, and we present only detailed results obtained with the former scenario.)

\subsection{Coherent neutrino scattering}

Coherent neutrino-nucleus elastic scattering is a predicted high-rate interaction of the Standard Model whereby neutrinos interact with the nucleus as a whole through $Z^{0}$ exchange [27]. This flavour-blind process measures the total neutrino flux and could have important practical applications (e.g. nuclear reactor monitoring) as well as probe new physics. For example, if this rate were observed to oscillate this could provide evidence for the existence of sterile neutrinos. Existing detectors exploit the elastic scattering of neutrinos off electrons or inelastic scattering from individual nucleons, in processes which produce much higher energy depositions (but lower rates) for neutrinos of the same energy. Several studies have proposed to attempt the extremely challenging detection of 
coherent neutrino scattering (e.g. [28-30]), and notably that in Ref. [7] addresses the type of signature studied here. In these studies, xenon has been consistently pointed out as a favourable detection medium when the combined effect of recoil energies and scattering rates is considered.

The maximum recoil energy, $E_{r}^{\max }$, from a neutrino with energy $E_{\nu}$ is inversely proportional to the mass of the target nucleus $(M)$ :

$$
E_{r}^{\max }=\frac{2 E_{\nu}^{2}}{M+2 E_{\nu}}
$$

The differential cross section is given by:

$$
\frac{d \sigma}{d E_{r}}=\frac{G_{F}^{2}}{4 \pi} Q_{W}^{2} M\left(1-\frac{M E_{r}}{2 E_{\nu}^{2}}\right) F^{2}\left(Q^{2}\right)
$$

where $G_{F}$ is the Fermi constant, $F\left(Q^{2}\right)$ is the form factor at four-momentum $Q$ and $Q_{W}=N-\left(1-4 \sin ^{2}\left(\theta_{W}\right)\right) Z \sim N$ is the weak charge for a nucleus with $N$ neutrons and $Z$ protons, with $\theta_{W}$ the weak mixing angle; $Q_{W}^{2}$ enhances the scattering rate through coherence and favours heavier elements.

\subsection{Signal rate estimation}

Assuming a recoil energy threshold $E_{t h}=0.5 \mathrm{keV}$, Eq.(3.1) places a lower bound of $5.5 \mathrm{MeV}$ on the detectable neutrino energy for a xenon target. On the other hand, maintaining scattering coherence requires small momentum transfer $(q R<1$, where $R$ is the nuclear radius and $q$ is the three-momentum), determining an upper bound of $\sim 50 \mathrm{MeV}$ for the neutrino energy. This range limits the neutrino sources that can be detected in this way. Seeking those with highest flux, we consider the following - represented in Figure 6:

- Solar neutrinos: a fraction of the solar neutrino spectrum, namely the ${ }^{8} \mathrm{~B}$ and 'hep' $\left({ }^{3} \mathrm{He}+\mathrm{p} \rightarrow{ }^{4} \mathrm{He}+\mathrm{e}^{+}+\nu_{\mathrm{e}}\right)$ contributions, delivers high flux in the interesting energy range. We assume the Bahcall model for the neutrino spectra [31]. (This case is included mainly for reference, since the scattering rates are very small indeed.)

- Reactor antineutrinos: we address the possibility of placing a detector like ZEPLINIII some $10 \mathrm{~m}$ away from a $3 \mathrm{GW}$ nuclear reactor, where the neutrino flux would be $\sim 4 \times 10^{13} \mathrm{~cm}^{-2} \mathrm{~s}^{-1}$; we adopt spectra from Refs. [32] and [33]. Several experiments have operated at similar distances from a nuclear reactor core and new ones are being considered [34-36].

- Stopped pion sources are promising for these studies [37]. We consider neutrinos produced at the $800 \mathrm{MeV}$ proton beam at the ISIS facility (Rutherford Appleton Laboratory), with a pulse repetition rate of $50 \mathrm{~s}^{-1}$ and beam current of $200 \mu \mathrm{A}$ [38]. The neutrino flux $10 \mathrm{~m}$ away from the target is estimated as $\sim 1 \times 10^{7} \mathrm{~cm}^{-2} \mathrm{~s}^{-1}$ per flavour. 


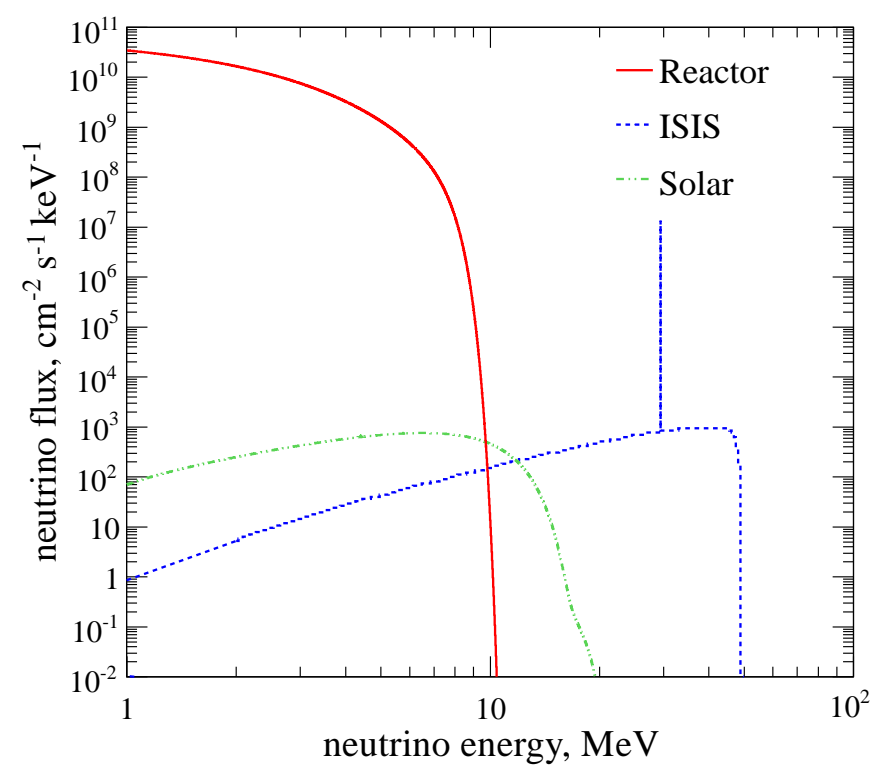

Figure 6. Neutrino fluxes considered in this work; the solar flux includes ${ }^{8} \mathrm{~B}$ and 'hep' contributions [31]; the reactor flux from Ref. [32] is adopted; the ISIS spectrum includes the beam-prompt $\nu_{\mu}$ line at $29.8 \mathrm{MeV}$ and the two continuum contributions from $\bar{\nu}_{\mu}$ and $\nu_{e}[38]$ (which follow a muon decay timescale with $\tau=2.2 \mu \mathrm{s})$.

The integral event rate is given by:

$$
R\left(E_{t h}\right)=N_{t} \int_{0}^{\infty} d E_{\nu} \Phi\left(E_{\nu}\right) \int_{E_{t h}}^{E_{r}^{\max }} d E_{r} \frac{d \sigma\left(E_{\nu}, E_{r}\right)}{d E_{r}},
$$

where $N_{t}$ is the number of target atoms, $\Phi\left(E_{\nu}\right)$ is the neutrino flux and $d \sigma / d E_{r}$ is the differential cross-section given by Eq.(3.2). Figure 7 shows the computed event rates of coherent neutrino-nucleus scatters in xenon for the neutrino fluxes mentioned above.

From these event rates the neutrino signal induced in the detector is evaluated. We consider $10 \mathrm{~kg} \cdot$ year as a reasonable dataset (1.5-year run with the nominal ZEPLIN-III fiducial mass of $6.5 \mathrm{~kg}$ ). For each neutrino-nucleus interaction, very few (if any) S1 VUV photons and some ionisation electrons would be generated, but the S1 signal is most likely lost. However, due to the sensitivity achieved in the ionisation channel, a measurement can be made even if only one electron is extracted from the interaction point. For S2-only events the depth information normally afforded by the time projection chamber is lost (but $x, y$ positional information is still available) and so is particle discrimination by $\mathrm{S} 2 / \mathrm{S} 1$ ratio. For this reason, it is essential to record S1 pulses whenever present; crucially, this will also help reject important backgrounds (e.g. radioactive contamination on the cathode grid).

\subsection{Backgrounds}

We assume that external radioactivity neutrons and those associated with the reactor or beam are suitably mitigated with sufficient hydrocarbon shielding combined with an anti- 


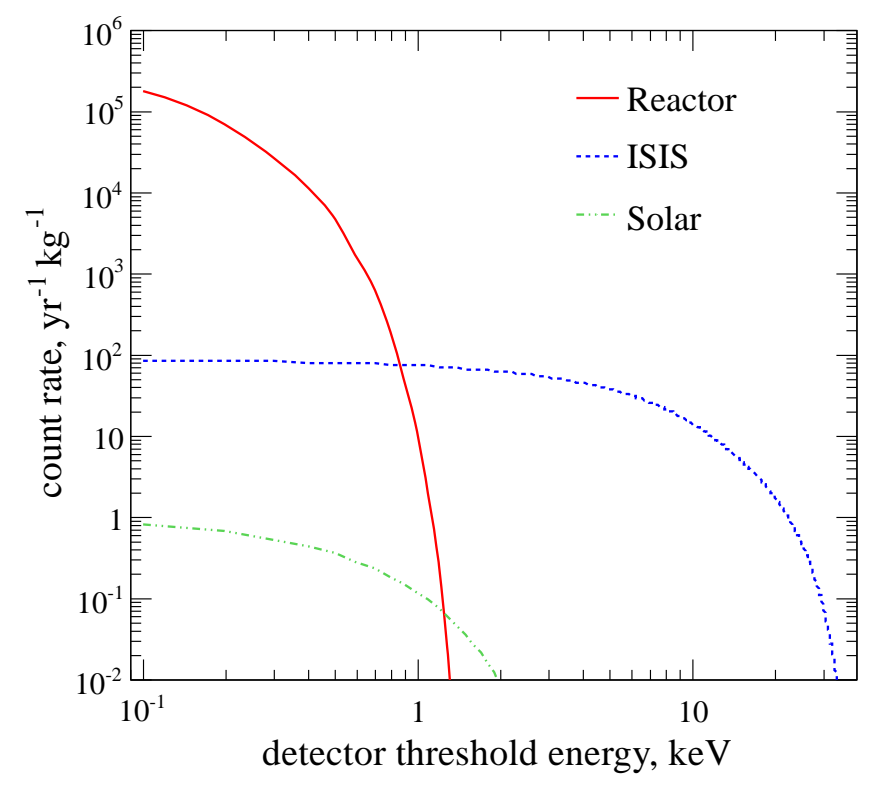

Figure 7. Expected integral rate of coherent neutrino-nucleus scatters in liquid xenon as a function of detector threshold for nuclear recoils.

coincidence system surrounding the xenon target - like that deployed in the second run of ZEPLIN-III [39, 40]. A veto detector will also tag internal neutrons efficiently and reduce the effect of cosmogenic neutrons to manageable levels.

A dominant background will arise from the single electron emission mechanisms discussed previously. We consider a rate $f \sim 10 \mathrm{~s}^{-1}$, which is comparable, per unit volume, to that observed (underground) for the SSR. Hopefully, a higher extraction field and a longer inhibit period to preceding events can mitigate the potentially higher event rate expected with a surface deployment. We include the probability of $n$-electron coincidences according to $n f^{n} \Delta t^{n-1}$, where $\Delta t=1 \mu \mathrm{s}$ is the typical single electron signal duration. We point out that the coincidences rate could be lowered significantly $(\sim 10$ for a detector like ZEPLIN-III) with more sophisticated position algorithms.

A more challenging background arises from low-energy electron recoils due to $\beta$ and $\gamma$ radiation (internal and cosmogenic, assuming substantial $\gamma$-ray shielding). The SSR background rate of $\sim 1$ event $/ \mathrm{kg} /$ day $/ \mathrm{keV}$, measured and modelled in Ref. [17], is considered. The average energy per emitted electron from an electron recoil is $\sim 50 \mathrm{eV}$ - obtained from the W-value for LXe at zero field [41] and relevant field-dependent extraction (from track) and surface emission probabilities. This background represents 180 events/electron in a $10 \mathrm{~kg} \cdot$ year dataset. Above $\sim 2 \mathrm{keV}$ in $\mathrm{S} 1$ (corresponding to $\sim 50$-electron $\mathrm{S} 2$ signals from electron recoils) efficient discrimination by $\mathrm{S} 2 / \mathrm{S} 1$ ratio will become possible.

This background can be suppressed significantly in an ISIS experiment by exploiting the pulsed nature of the beam: a triggered measurement lasting for one drift length $(\sim 20 \mu \mathrm{s})$ per proton bunch (20 ms period) would effectively reduce most non beam-related 
backgrounds by a factor of $\sim 1,000$. In addition, the depth coordinate can still be recovered with reasonable accuracy by replacing the $\mathrm{S} 1$ pulse by the trigger signal, enabling fiducialisation and self-shielding of external backgrounds - this will work well for the beam-prompt neutrinos; the continuum components will be delayed by a few $\mu$ s, reducing the timing resolution of the chamber. For a reactor experiment, 'on/off' background subtraction will be far less effective.

\subsection{Predicted observable spectra}

In Figure 8 we present photoelectron spectra predicted for neutrino interactions and for the two dominant backgrounds, as would be observed in ZEPLIN-III. Individual peaks represent ionisation electrons detected by electroluminescence; we assume a yield of 30 photoelectrons per electron and Poisson variance.

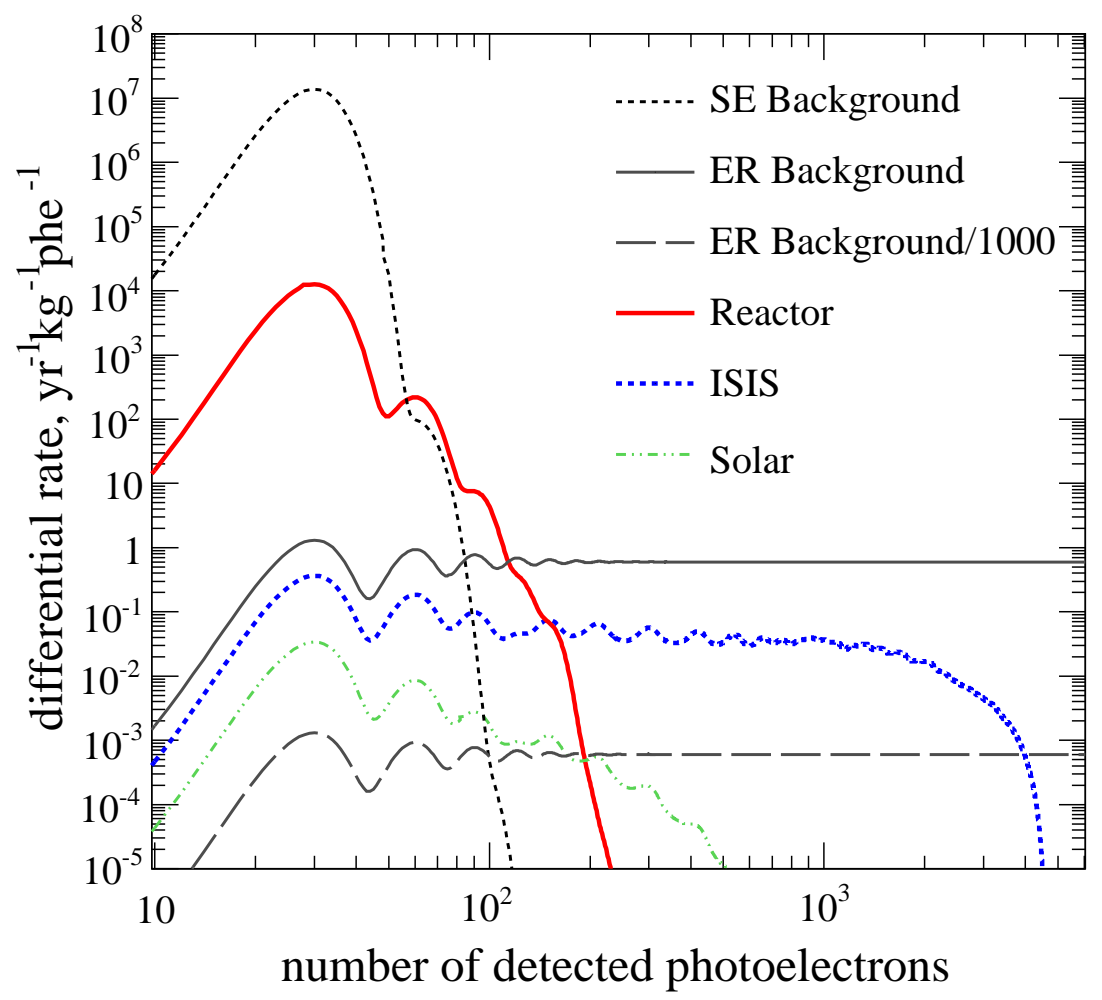

Figure 8. Ionisation spectra expected from coherent neutrino scattering in ZEPLIN-III exposed to different neutrino sources. Single electron and electron recoil backgrounds are also shown. The peak structure reflects discrete numbers of ionisation electrons measured by electroluminescence.

As the figure suggests, the neutrino signal must be searched above $\gtrsim 3$ electrons due to the single electron background - although this will be improved with multiple-cluster resolution in $x, y$ using advanced position algorithms. The electron recoil background becomes significant above that threshold, but the reactor signal is clearly salient near 100 phe. Unfortunately, its spectrum does not extend to 1,500 photoelectrons in the S2 
channel (50 electrons), which would be required for a detectable S1 pulse from an electron recoil thus enabling discrimination by $\mathrm{S} 2 / \mathrm{S} 1$ ratio.

For a reactor experiment, the number of events expected in a $10 \mathrm{~kg} \cdot \mathrm{yr}$ dataset above a 75 phe threshold is of order 3,000 (1,000 above 90 phe). The electroninc background is $\sim 200$ events over the relevant range. These values are sensitive to the shape of the antineutrino reactor spectrum and the ionisation yield for low energy recoils. The number of signal events changes only by a few tens of percent when the antineutrino spectrum of Ref. [33] is used instead. However, the optimistic ionisation yield scenario mentioned previously would increase the signal rate ten-fold. This is therefore an appropriate level of systematic uncertainty to frame these calculations as far as the signal is concerned. The background in the reactor environment will depend critically on the shielding efficiency, which must be higher than typically required for underground WIMP searches. We note also that self-shielding will not be very effective in the absence of the depth coordinate. In spite of this, the reactor experiment seems viable.

Signal rates at the stopped pion source are lower but the spectrum does extend to considerable energies. Significantly, in this instance the electron recoil background can be reduced by three orders of magnitude with a beam-coincident measurement. Discrimination should be possible for approximately half of the events, when S1 pulses are expected. 3D position reconstruction should be achieved to a resolution of a few $\mathrm{mm}$ in the depth coordinate by using the trigger signal to define zero drift time. The integrals above 75 phe are $\sim 700$ and $\sim 10$ background events over the same energy range for a $10 \mathrm{~kg} \cdot$ year exposure. This result is not very sensitive to the ionisation yield at low energy. In conclusion, the spallation source produces very encouraging numbers.

\section{Conclusions}

In this article we report studies of single electron emission using data from the ZEPLIN-III dark matter experiment and assess two applications which exploit the ability of xenon emission detectors to sense the quantum of response in ionisation. Electroluminescence signals due to single electrons emitted from the liquid xenon target were analysed. We showed that such pulses, containing an average of $\sim 30$ photoelectrons in the FSR configuration, can be detected with very high signal-to-noise ratio and exhibit near-Poisson variance.

The source of single electrons following the scintillation generated by sizable energy depositions in the liquid xenon is thought to be photoionisation of impurities by the VUV photons. We demonstrated that this signature can be used to obtain a very robust measurement of the free electron lifetime in the liquid phase from science data themselves.

In addition to this photon-induced population, 'spontaneous' electron emission was also studied and attributed to delayed emission of thermal electrons trapped at the surface. Clearly, the physics of the emission process at the liquid-gas interface is a topic deserving further study. It was also concluded that field emission from cathode wire grids could be significant and should be considered when designing these detectors. These single electron pulses determine a $\sim 3$ electron threshold for experiments exploiting the ionisation channel below the scintillation threshold. 
We assessed the feasibility of two such experiments related to the detection of coherent neutrino-nucleus elastic scattering, a Standard Model process not yet observed, using realistic signal characteristics and backgrounds. We found that the signal from the nuclear reactor scenario considered in this study is salient above the electron recoil background, with $\gtrsim 2,000$ events $/(10 \mathrm{~kg} \cdot \mathrm{yr})$ expected above a $\sim 3$-electron threshold. In this instance the signal rate is very sensitive to the nuclear recoil ionisation yield assumed at low energies.

The prospects of detecting this elusive neutrino signature with a beam-coincident measurement at a stopped pion source such as ISIS are also very encouraging: owing to a harder energy spectrum, a sizable fraction of events should benefit from electron-nuclear recoil discrimination; three-dimensional position reconstruction should be possible; backgrounds would be significantly lower. $\gtrsim 700$ events/(10 kg.yr) are expected above $\sim 3$ emitted electrons. Even higher neutrino fluxes are likely to be reached in the coming years: SNS at Oak Ridge [42] is expected to achieve a flux several times higher than that considered here, and ISIS itself may have its capability improved in a forthcoming upgrade [43].

\section{Acknowledgments}

The UK groups acknowledge the support of the Science \& Technology Facilities Council (STFC) for the ZEPLIN-III project and for maintenance and operation of the Boulby underground laboratory. LIP-Coimbra acknowledges support from Fundação para a Ciência e a Tecnologia (FCT) through project grant CERN/FP/116374/2010 and postdoctoral grants SFRH/BPD/27054/2006, SFRH/BPD/47320/2008 and SFRH/BPD/63096/2009. The ITEP group acknowledges support from the Russian Foundation of Basic Research (grant 08-02-91851 KO_a) and Rosatom (H.4e.45.90.11.1059 from 10-03-2011). We are also grateful for support provided jointly to ITEP and Imperial from the UK Royal Society. ZEPLIN-III is hosted by Cleveland Potash Ltd (CPL) at the Boulby Mine and we thank CPL management and staff for their long-standing support. We also express our gratitude to the Boulby facility staff for their dedication. The University of Edinburgh is a charitable body registered in Scotland (SC005336).

\section{References}

[1] T. J. Sumner in Identification of Dark Matter (Spooner \& Kudryavtsev, ed.), p. 452, 2001.

[2] D. Y. Akimov et. al. Astropart. Phys. 27 (2007) 46.

[3] B. A. Dolgoshein, V. N. Lebedenko, and B. U. Rodionov JETP Lett. 11 (1970) 351.

[4] A. Barabash and A. Bolozdynya JETP Lett. 49 (1989) 356.

[5] A. Bolozdynya et. al. IEEE Trans. Nuc. Sci. 42 (1995) 565.

[6] A. Fonseca et. al. IEEE Trans. Nuc. Sci. 1 (2004) 572.

[7] C. Hagmann and A. Berstein IEEE Trans. Nucl. Sci. 51 (2004) 2151.

[8] S. Sangiorgio et. al. in IEEE NSS Conf. Rec. 2010, p. 102, 2010.

[9] J. Angle et. al. Phys. Rev. Lett. 107 (2011) 051301. 
[10] B. Edwards et. al. Astropart. Phys. 30 (2008) 54.

[11] A. A. Burenkov et. al. Phys. At. Nucl. 72 (2009) 653.

[12] F. Neves et. al. arXiv:1106.0808 (2011).

[13] V. N. Lebedenko et. al. Phys. Rev. D 80 (2009) 052010.

[14] V. N. Lebedenko et. al. Phys. Rev. Lett. 103 (2009) 151302.

[15] D. Y. Akimov et. al. Phys. Lett. B 692 (2010) 180.

[16] H. M. Araújo et. al. Nucl. Instrum. Meth. A 521 (2004) 407.

[17] H. M. Araújo et. al. arXiv:1104.3538 (2011).

[18] E. Gushchin et. al. Sov. Phys. JETP 49 (1979) 856.

[19] A. I. Bolozdynya Nucl. Instrum. Meth. A 422 (1999) 314.

[20] R. J. Noer Appl. Phys. A 28 (1982) 1.

[21] B. M. Cox and W. T. Williams J. Phys. D: Appl. Phys. 10 (1977) L5.

[22] E. Gordon, O. Rzhevskii, and V. Khmelenko Quantum Electron. 24 (1994) 209.

[23] R. Veenhof, Garfield - simulation of gaseous detectors, 1984. CERN Prog. Lib., W5050.

[24] R. Fowler and L. Nordheim Proc. R. Soc. Lond A 119 (1928) 173.

[25] M. Horn et. al. arXiv:1106.0694 (2011).

[26] A. Manzur et. al. Phys. Rev. C 81 (2010) 025808.

[27] D. Freedman Phys. Rev. D 9 (1974) 1389.

[28] J. Collar J. Phys.: Conf. Ser 173 (2009) 012022.

[29] K. Scholberg et. al. arXiv:hep-ex/0910.1989v2 (2009).

[30] A. Anderson et. al. prd 84 (2011) 013008.

[31] J. Bahcall, M. Pinsonneault, and G. J. Wasserburg Rev. Mod. Phys. 67 (1995) 781.

[32] V. Kopeikin et. al. Phys. At. Nuc. 60 (1997) 172.

[33] P. Vogel and J. Engel Phys. Rev. D 39 (1989) 3378.

[34] M. Avenier Nucl. Instrum. Meth. A 284 (1989) 29.

[35] A. Porta J. Phys.: Conf. Ser. 203 (2010) 012092.

[36] D. Akimov et. al. J. Instrum. 4 (2009) P06010.

[37] K. Scholberg Phys. Rev. D 73 (2006) 033055.

[38] B. Armbruster et. al. Nucl. Phys. A 629 (1998) 507.

[39] D. Y. Akimov et. al. Astropart. Phys. 34 (2010) 151.

[40] C. Ghag et. al. Astropart. Phys. 35 (2011) 76.

[41] T. Takahashi et. al. Phys. Rev. A 12 (1975) 1771.

[42] Y. Efremenko and W. R. Hix J. Phys.: Conf. Ser. 173 (2009) 012006.

[43] J. Thomason et. al., Megawatt upgrades for the ISIS facility, in Proc. EPAC'08 - 11th European Particle Accelerator Conference, p. 3554, 2008. 